Article

\title{
Tenskinmetric Evaluation of Surface Energy Changes in Adult Skin: Evidence from 834 Normal Subjects Monitored in Controlled Conditions
}

\author{
Camilla Dal Bosco ${ }^{1}$, Davide Rossi ${ }^{1}$, Andrea Brunetta ${ }^{2}$ and Antonio Bettero ${ }^{1, *}$ \\ 1 Department of Pharmaceutical and Pharmacological Sciences, University of Padova, Via Marzolo 5, \\ 35131 Padova, Italy; E-Mails: camilla.dalbosco@unipd.it (C.D.B.); davide.rossi@unipd.it (D.R.) \\ 2 Kalis srl, Via Caodevilla 38, Onigo di Pederobba 31040, Treviso, Italy; \\ E-Mail: andrea.brunetta@kalis.it
}

* Author to whom correspondence should be addressed; E-Mail: antonio.bettero@unipd.it;

Tel.: +39-049-827-5335; Fax: +39-049-827-5366.

Received: 12 January 2014; in revised form: 24 February 2014 / Accepted: 24 February 2014 / Published: 4 March 2014

\begin{abstract}
To evaluate the influence of the skin aging critical level on the adult skin epidermal functional state, an improved analytical method based on the skin surface energetic measurement (TVS modeling) was developed. Tenskinmetric measurements were carried out non-invasively in controlled conditions by contact angle method using only a water-drop as reference standard liquid. Adult skin was monitored by TVS Observatory according to a specific and controlled thermal protocol (Camianta protocol) in use at the interconnected "Mamma Margherita Terme spa" of Terme Euganee. From June to November 2013, the surface free energy and the epidermal hydration level of adult skin were evaluated on arrival of 265 male and 569 female adult volunteers (51-90 years of age) and when they departed 2 weeks later. Sensitive measurements were carried out at $0.1 \mathrm{mN} / \mathrm{m}$. High test compliance was obtained (93.2\% of all guests). Very interesting results are obtained. The high sensitivity and discrimination power of tenskinmetry combined with a thermal Camianta protocol demonstrate the possibility to evaluate at baseline level the surface energetic changes and the skin reactivity which occurs on adult skin.
\end{abstract}

Keywords: tenskinmetry; TVS skin test; contact angle method; Camianta protocol 


\section{Introduction}

Tenskinmetry is a conceptually innovative TVS pathway for non-invasive evaluation of surface energy phenomena closely related to the epidermal functional state.

TVS (Tensiometric Versus Skin) modeling (Figure 1): (i) is based on the centrality of the skin as presumed and essential knowledge; (ii) exploits the structure-surface correlations which are characteristic of all systems; (iii) applies the principle of permutability of the tensiometric technique, according to which unknown solids can be characterized by their known surface characteristics, and vice versa; (iv) applies the contact angle method, with only water as reference liquid; (v) is carried out with the tenskinmeter directly in contact with the skin in a non-invasive way; (vi) measures and correlates the surface free energy reflex induced by inter- and intra-molecular and particle forces acting on underlying epidermic layers [1-9].

Figure 1. TVS modeling.

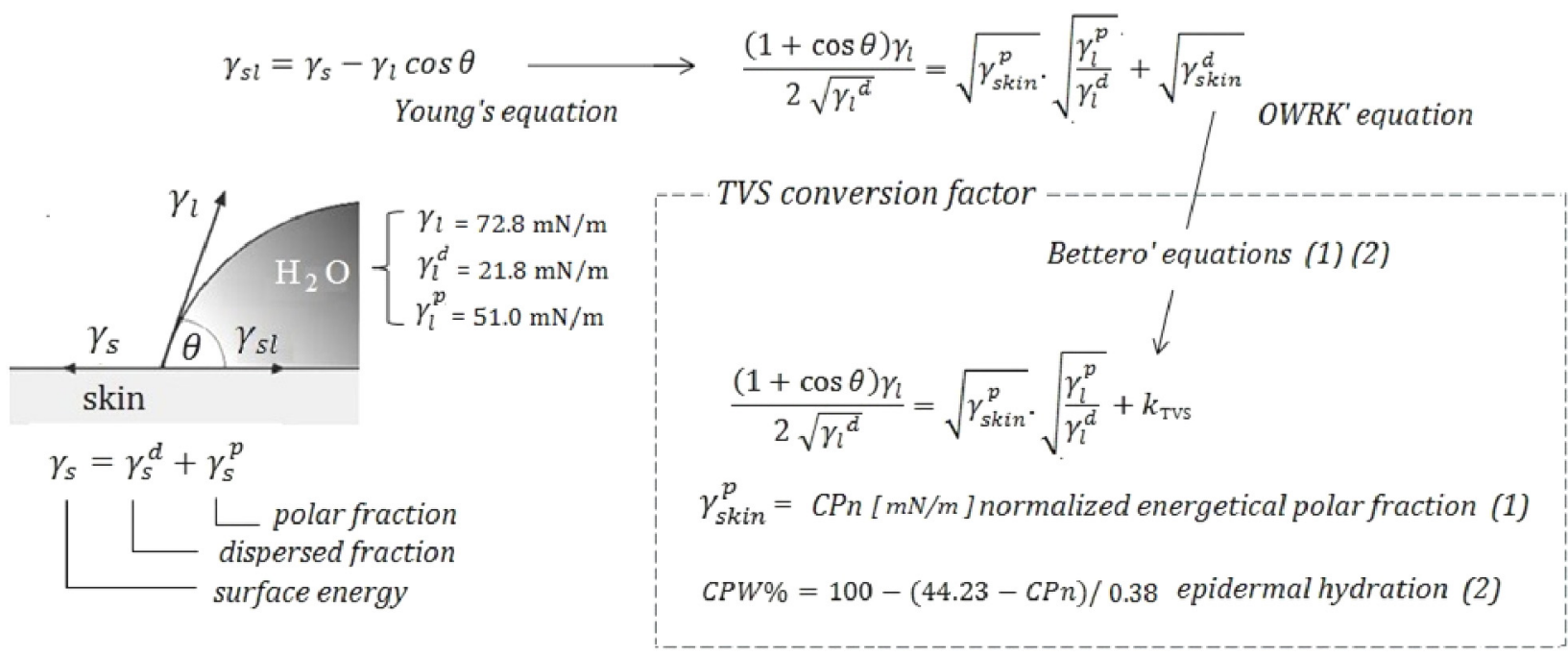

The contact angle formed by a drop of water on the skin is closely related to the polar energy reflex of the skin and consequently to its level of epidermal hydration [4].

The tenskinmeter used here was a combined MobileDrop GH11-TVS system developed for skin measurements (KRÜSS GmbH, Amburg, Germany).

TVS modeling involves the TVS skin test (forearm and skin mapping) for diagnostic purposes and Topical Design TVS for technological purposes (Table 1).

The TVS skin test uses water as a biocompatible liquid and a specific normalized liposome dispersion as biomimetic activator [4]. The test measures the surface free energy state of the skin, its hydration level, and reactivity after biomimetic activation.

In the period 2000-2013, the TVS Observatory of the University of Padova performed more than 3200 large-scale TVS tests in various conditions, developing significant TVS energy skin nomograms for each skin type, gender and age of subject.

To evaluate on a large scale the influence of the aging critical level on the epidermal function efficiency and selectivity in adult skin by a Camianta thermal protocol, two repeated TVS skin tests in controlled conditions were performed. 
Table 1. The non invasive contact angle measurement by TVS skin test.

Tenskinmeter Esplicative forearm and skin mapping tenskinmeter positioning

Camianta is the acronym of a TVS applicative protocol indicating the synergic combination of a Topical Design TVS for technological purposes with a TVS skin test for diagnostic and evaluation purposes.

In euganean thermal area, a specific Camianta thermal protocol was developed by combining the action of mature natural fango (TVS mud index controlled) with TVS skin test evaluation before and after its application.

From 2011, at Mamma Margherita Terme spa (HMMT) of Terme Euganee, Camianta thermal protocols were monitored by the TVS Observatory.

To evaluate the surface energy changes with occurs on adult skin, Camianta monitoring on a large scale were then performed by two repeated TVS skin tests on arrival and departure at the spa center.

From June to November 2012, 265 male and 569 female volunteers (age range: 51-90) were monitored.

\section{Experimental Section}

All guests who had already booked a two-week period, on arrival submitted to a medical examination, to check their suitability for spa treatment, and those who wished to enter the Camianta protocol did so on a volunteer basis. In controlled conditions, and with simultaneous computerized reporting, the TVS skin test was carried out on the left forearm of all volunteers, the right forearm being used as a control.

As a biomimetic activator, a specific biocompatible multilamellar liposomial spray dispersion was employed (by Kalis srl, Onigo di Pederobba-Treviso, Italy). The test was repeated on the day of guests' departure.

Camianta is based on an initial TVS skin test $\left(\mathrm{T}_{0}\right)$ to assess the basal energy level of the skin $(\mathrm{CPn})$, its degree of hydration (CPW\%) and reactivity (Rs) after biomimetic activation (Figure 2). This was followed by a second TVS test without biomimetic activation, for objective evaluation of the influence and efficacy of a specific health treatment.

All reported data are expressed as the mean plus standard deviation of five measurements.

Figure 2. Camianta protocol.

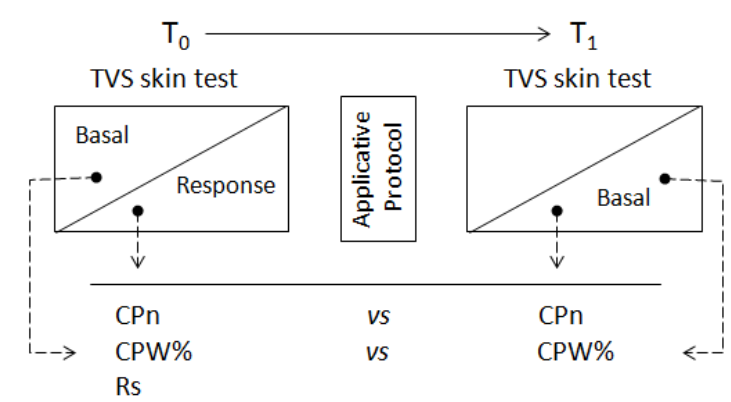




\section{Results and Discussion}

Preliminarily, a panel carried out to ascertain the applicability and practicality of the TVS skin test on adult skin even in very old subjects, with unexpected results as regards compliance: in fact, $93.2 \%$ of guests voluntarily underwent the Camiana protocol because of its non-invasiveness and the fact that only one drop of water could provide objective responses on the aging critical level and the possibility of functional skin recovery due to a biomimetic activator.

A total of 834 Camianta protocols was thus carried out on a sample population mainly composed of women (569) subjects, average age 68 years, and 265 men, mean age 70, distributed equally, from June to November 2012 (Figure 3).

The frequency of arrivals is typical of the spa season in the Euganean hotels and health centers, which mainly focus on mud therapy.

At HTMM, therapy is carried out with mature mud with a controlled mineralogico-chemical titer and tensiometricprint (TVS mud index $=59.4 \pm 6.2 \mathrm{mN} / \mathrm{m}$ ) [10].

Figure 3. Guest arrivals by gender.

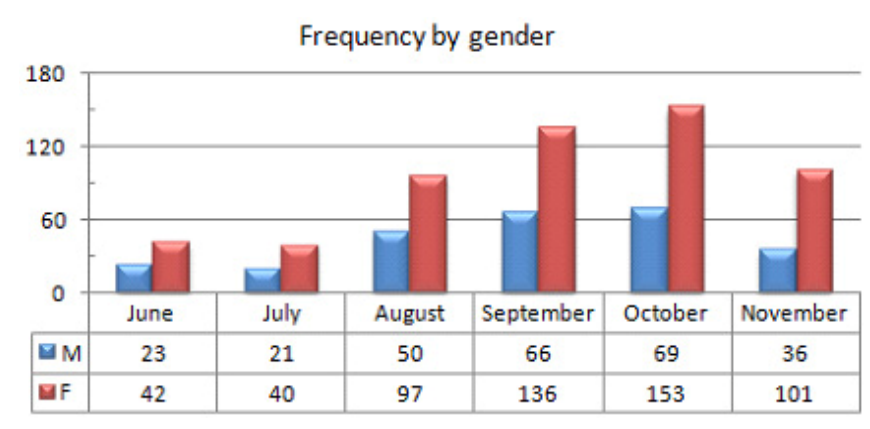

Figure 4 shows frequency by age group. Note the significant number of guests over 80 years old.

Figure 4. Guest distribution by age group.

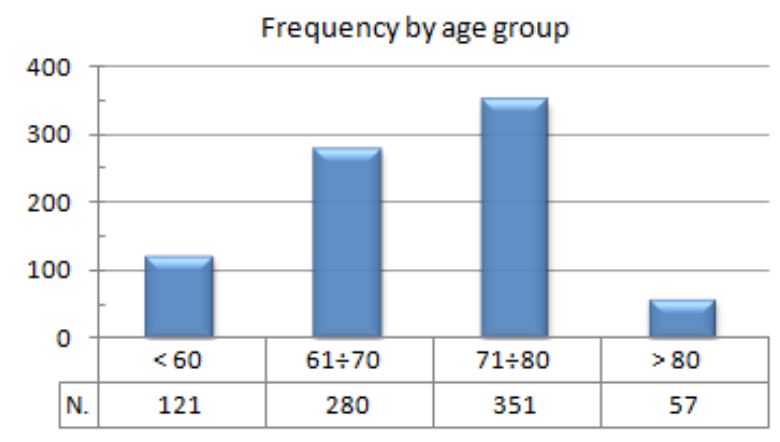

Figure 5 shows the sampled basal energy levels and those induced by the Camianta protocol: note the definite increase in terms of functional energy recovery, which was CPn $37.5 \%$.

The data listed in Tables 2 and 3 show the definite increase in skin energy (CPn) and epidermal hydration (CPW\%) in all age groups. By Dixon's $Q$-test $59.52 \%$ of $\Delta \mathrm{CPn}$ data demonstrated to be significatively discriminated. 
Figure 5. Surface energy changes in adult skin after treatment with Camianta protocol.

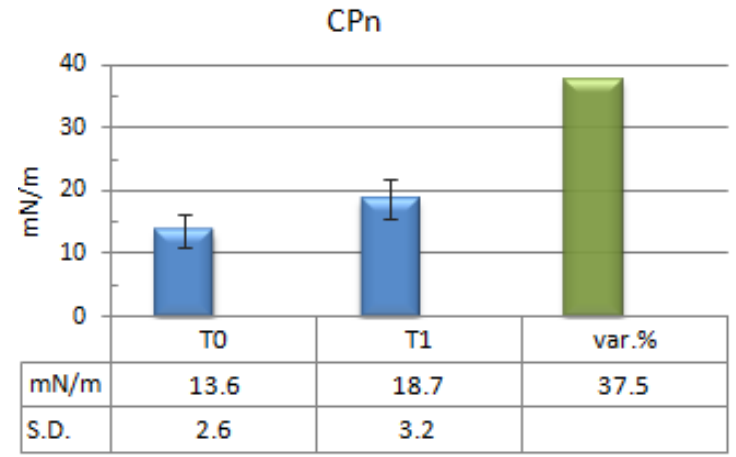

Table 2. Recovery of surface skin energy.

\begin{tabular}{ccccc}
\hline \multirow{2}{*}{ Age years } & \multirow{2}{*}{ Frequency $(\%)$} & \multicolumn{2}{c}{$\mathbf{C P n}(\mathbf{m N} / \mathbf{m})$} & \multirow{2}{*}{ Var.\% } \\
\cline { 3 - 5 } & & $\mathbf{T}_{\mathbf{0}}$ & $\mathbf{T}_{\mathbf{1}}$ & \\
\hline$<60$ & 15.0 & 13.5 & 19.5 & 44.2 \\
$61-70$ & 34.6 & 13.3 & 18.1 & 36.4 \\
$71-80$ & 46.8 & 13.8 & 18.9 & 37.2 \\
$>80$ & 7.3 & 14.7 & 19.7 & 34.0 \\
\hline
\end{tabular}

Table 3. Recovery of epidermal hydration.

\begin{tabular}{ccccc}
\hline \multirow{2}{*}{ Age years } & \multirow{2}{*}{ Frequency (\%) } & \multicolumn{2}{c}{ CPW\% (\%) } & \multirow{2}{*}{ Var.\% } \\
\cline { 3 - 4 } & & $\mathbf{T}_{\mathbf{0}}$ & $\mathbf{T}_{\mathbf{1}}$ & \\
\hline$<60$ & 15.0 & 18.8 & 34.7 & 84.3 \\
$61-70$ & 34.6 & 18.2 & 31.0 & 70.5 \\
$71-80$ & 46.8 & 19.5 & 33.0 & 69.7 \\
$>80$ & 7.3 & 22.0 & 35.1 & 59.2 \\
\hline
\end{tabular}

As Figure 6 shows, on arrival, the specific skin reactivity (Rs) of subjects under 80 showed lower basal values compared with those in the younger age groups, although the basal energy level was higher, after taking account of age.

Figure 6. Influence of age group on skin reactivity.

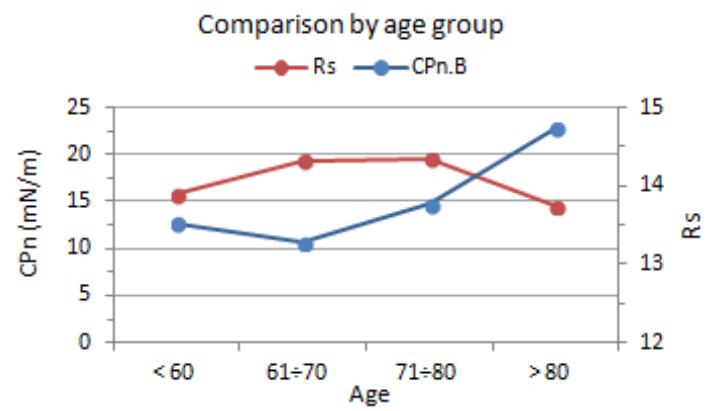

As an example, Figure 7 compares the basal energy levels, specific skin reactivity and functional skin recovery of younger subjects with respect to older ones. However, compared with the basal energy value, note the immediate specific reactivity of older subjects' skin in relation to the different critical limit of skin aging. 
Figure 7. Typical example of age on recovery of functional energy.

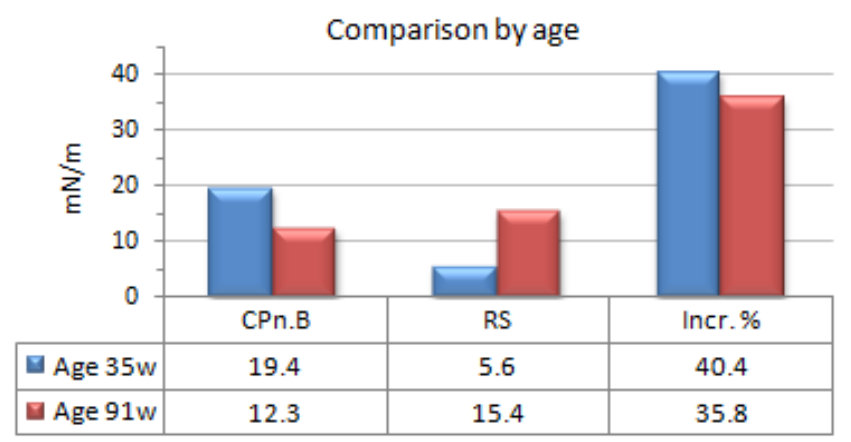

\section{Conclusions}

The influence of the aging critical level on the epidermal functional state and skin reactivity in adult skin can be objectively investigated by TVS modeling via TVS skin test.

The high sensitivity and discrimination power of tenskinmetry allows us to evaluate in a non invasive way the surface energy changes which occur in adult skin by the loss of the epidermal barrier function efficiency due to the reduced biosynthetic activity of the epidermal granular layer.

Combined with a TVS Camianta protocol, is possible to evaluate, by tenskinmetry, at baseline level the adult skin reactivity and its functionality recovery for age.

For the first time on a large scale, high TVS skin test compliance was obtained.

Thanks to its biocompatibility and high surface free energy profile, water as a reference liquid enabled contact angle measurements to be performed without any side-effects on any type of skin, even in aged persons. Very interesting results were obtained on a large scale in the present study.

In order to discriminate for age when considering the surface energetic changes induced in old skin monitored by the Camianta thermal protocol, the multivariate analysis of the data is in progress.

\section{Acknowledgments}

We thank Massimiliano Catto director, Nicola Mazzacuva and Enzo Michelon (H. Mamma Margherita Terme, Terme Euganee) Paolo Colombini (Elith srl, Trento-Italy). We also thank Margherita Bottacin, Chiara Chieregato and Luisa Matteoli for TVS skin test monitoring.

\section{Author Contributions}

Camianta evaluate the data, and reports were performed by Camilla Dal Bosco.

\section{Conflicts of Interest}

The authors declare no conflict of interest.

\section{References}

1. Owens, D.; Wendt, R. Estimation of the surface free energy of polymers. J. Appl. Polym. Sci. 1969, 13, 1741-1747. 
2. Kaelble, D.H. Dispersion-polar surface tension properties of organic solids. J. Adhes. 1970, 2, 66-81.

3. Rabel, W. Einige Aspekte der Benetzungstheorie und ihre Anwendung auf die Untersuchung und Veränderung der Oberflächeneigenschaften von Polymeren. Farbe und Lack 1971, 10, 997-1005. (In German).

4. Dal Bosco, C. Evaluation of Skin Moisturizing and of Its Modulations by TVS Skin Test. Ph.D. Thesis, University of Ferrara, Padova, Milano, Italy, 2014.

5. Dal Bosco, C.; Rossi, D.; Bettero, A. Introduction to the "topical design TVS". In Proceedings of 5th World Meeting on Pharmaceutics Biopharameceutics \& Pharmaceutical Technology, Geneva, Switzerland, 1-2 March 2006; pp. 1-3.

6. Bettero, A.; Dal Bosco, C.; Rossi, D.; Mignini, E. Introduction to bioadhesive topical design TVS: A conceptually innovative pathway in the design phase, development and efficacy evaluation of cosmetic products. In Proceedings of Cosmeticos, Tecnologia e Meio Ambiente Proc. ABC Int. Congress, San Paolo, Brasil, 27-29 May 2008; pp. 1-3.

7. Rossi, D.; Bettero, A.; Dal Bosco, C. Tensiometric versus skin (TVS) modeling as improved approach for the direct evaluation of skin hydration. In Proceedings of Frontiers in Water Biophysics International Conference, Trieste, Italy, 23-26 May 2010; pp. 23-26.

8. Bettero, A.; dal Bosco, C.; Rossi, D.; dal Bosco, Ch. Correlation between epidermal tihickening and surface free energy reflex in patients operated for breast carcinoma by a combined ultrasound imaging and tenskinmetric analysis (TVS skin test). The Influence of a Biomimetic Cosmetic-Treatment. In Proceedings of Innovation and Responsability Cosmetic Forever, 26th IFSCC International Congress, Buenos Aires, Argentine, 21-24 September 2010; pp. 21-24.

9. Dal Bosco, C.; Bettero, A. Evaluation of skin affinity and cosmetic functionality of nanoparticle systems by TVS skin test. In Proceedings of Nanotechnologies and Cosmetics, NUCE International, Milano, Italy, 26 October 2010.

10. Rossi, D.; Jobstraibizer, P.G.; dal Bosco, C.; Bettero, A. A combined chemico-mineralogical and tensiometric approach for evaluation of Euganean Thermal Mud (ETM) qualty. J. Adhes. Sci. Technol. 2013, 27, 30-45.

(C) 2014 by the authors; licensee MDPI, Basel, Switzerland. This article is an open access article distributed under the terms and conditions of the Creative Commons Attribution license (http://creativecommons.org/licenses/by/3.0/). 